\title{
Comments on Magnetic Reconnection Models of Canceling Magnetic Features on the Sun
}

\author{
Yuri E. Litvinenko \\ Department of Mathematics, University of Waikato, P. B. 3105, Hamilton, New Zealand; yuril@waikato.ac.nz \\ Received February 6, 2015; accepted May 18, 2015
}

\begin{abstract}
Data analysis and theoretical arguments support magnetic reconnection in a chromospheric current sheet as the mechanism of the observed photospheric magnetic flux cancellation on the Sun. Flux pile-up reconnection in a Sweet-Parker current sheet can explain the observed properties of canceling magnetic features, including the speeds of canceling magnetic fragments, the magnetic fluxes in the fragments, and the flux cancellation rates, inferred from the data. It is discussed how more realistic chromospheric reconnection models can be developed by relaxing the assumptions of a negligible current sheet curvature and a constant height of the reconnection site above the photosphere.
\end{abstract}

Key words: magnetic reconnection — Sun: chromosphere - Sun: magnetic fields — Sun: photosphere

\section{INTRODUCTION}

Video magnetograms of the photospheric magnetic field on the Sun often show that magnetic fragments with opposite polarity approach each other and disappear. The observational term "cancellation" describes the disappearance of magnetic flux of either sign in such canceling magnetic features at the polarity inversion line that separates the fragments (Livi et al. 1985; Martin et al. 1985). Photospheric magnetic flux cancellation is interesting both in its own right and as a key process in the formation and evolution of solar filaments (Martens \& Zwaan 2001; Martin et al. 2008; Chae 2012; Panasenco et al. 2014). Photospheric cancellation has been studied using the data from instruments on board several satellites, including Solar and Heliospheric Observatory (Chae et al. 2002), Hinode (Park et al. 2009), and Solar Dynamics Observatory (Zeng et al. 2014).

Several observational arguments strongly suggest that magnetic reconnection in a photospheric or chromospheric current sheet, rather than simple submergence, is the cancellation mechanism (Martin 1990). First, magnetic fragments originate as bipoles but cancel with external fields. Second, fragments with the same polarity do not cancel on encounter but rather merge to form a single larger magnetic feature. Third, canceling fragments slow down on encounter, indicating that mutual interaction takes place. On the theoretical side, magnetic reconnection can explain the observed properties of canceling magnetic features. Specifically, predictions of a model of flux pile-up reconnection in a Sweet-Parker current sheet agree with the observed speeds of canceling magnetic fragments, the magnetic fluxes in the fragments, and the flux cancellation rates, inferred from the data (Litvinenko 1999; Litvinenko et al. 2007).

The above-cited reconnection models adopt a simplified cancellation geometry: the current sheet curvature

Corresponding AUthor: Y. E. Litvinenko is assumed to be negligible, and the height of the reconnection site above the photosphere is assumed to be independent of time. The purpose of this note is to discuss how these assumptions could be relaxed, so that more realistic models of canceling magnetic features could be developed.

\section{Magnetic Merging in a Curved CurRent SheET}

In the context of magnetic reconnection modeling of photospheric cancellation, it is significant that exact analytical solutions are available, which illustrate the key features of magnetic reconnection, including a small thickness of the current sheet, flux pile-up at the entrance to the sheet, and Alfvénic outflows. Traditional flux pile-up models describe magnetic merging (annihilation) or reconnection in a flat current sheet, sustained by a stagnation-point flow in two or three dimensions.

Litvinenko (2013) has recently presented a magnetohydrodynamic solution for magnetic merging in a curved current sheet, driven by a three-dimensional stagnation flow. Below the solution for the velocity $\mathbf{v}$ and magnetic field $\mathbf{B}$ in an incompressible resistive plasma is presented in dimensionless form by adopting reference values of the magnetic field $B_{0}$, plasma density $\rho_{0}$, and length $L$. The speed is measured in units of the Alfvén speed $v_{A}=B_{0} / \sqrt{4 \pi \rho_{0}}$, and the dimensionless parameter $\eta$ is the plasma resistivity, normalized by $4 \pi v_{A} L / c^{2}$, where $c$ is the speed of light. For simplicity, viscosity and time-dependence are not considered.

The steady merging solution (Litvinenko 2013) is based on an axisymmetric stagnation flow on a cylinder. In polar coordinates, the velocity is given by

$$
\mathbf{v}=-\alpha\left(r-\frac{a^{2}}{r}\right) \hat{\boldsymbol{r}}+2 \alpha z \hat{\boldsymbol{z}},
$$

where $\alpha$ and $a$ are constants. The singularity at the origin corresponds to a source if $\alpha>0$ and a sink if 
$\alpha<0$. When $a>0$, the flow stagnation takes place on a circle of radius $a$ which is the intersection of the $x y$-plane and a circular cylinder whose generators are parallel to the $z$-axis. In the limit $a=0$, the flow reduces to the stagnation-point flow. Assuming $\alpha>0$, the merging magnetic field is given by

$$
\mathbf{B}=B(r) \hat{\boldsymbol{z}}
$$

where

$$
\begin{gathered}
B(r)=r^{\alpha a^{2} / \eta} \exp \left(-\frac{\alpha r^{2}}{2 \eta}\right) f(r), \\
f(r)=a J \int r^{-1-\alpha a^{2} / \eta} \exp \left(\frac{\alpha r^{2}}{2 \eta}\right) d r .
\end{gathered}
$$

Here $J=B^{\prime}(a)$ is the dimensionless electric current density at $r=a$. To describe magnetic merging in the flow stagnation region, an integration constant should be chosen so that $B(a)=0$, in which case a curved current sheet is located at $r=a$.

The solution is formally valid in any range of the azimuthal angle. In practice, it makes sense to consider the solution in a finite annular region. If the curvature of the current sheet is negligible, $|1-r / a| \ll 1$, the solution for merging in a flat sheet is recovered (Craig \& Henton 1995).

If the dimensionless current sheet thickness $l \ll 1$, a boundary-layer argument yields a simple scaling

$$
l \approx\left(\frac{\eta}{2 \alpha}\right)^{1 / 2}
$$

and the magnetic field $B_{s}$ at the entrance to the sheet follows from

$$
B_{s} \approx J l
$$

(Litvinenko 2013). Thus the magnetic merging scalings are the same for a curved current sheet and for a flat one. The new solution extends the earlier results, derived in a planar geometry (Craig \& Henton 1995) and emphasizes the key requirement of flux pileup $\left(B_{s}>1\right)$ in order for the merging rate to exceed the Sweet-Parker rate $\simeq \eta^{1 / 2}$. Fast merging, characterized by $\eta J \simeq 1$, can be achieved in a current sheet with $B_{s} \simeq l / \eta \sim \eta^{-1 / 2}$.

The solution for merging in a curved current sheet may be particularly useful in modeling the photospheric flux cancellation on the Sun. The magnetic field in the above-given merging solution is perpendicular to the plane $z=0$ in which the flow stagnates. The plane can be identified with the solar photosphere and the field with the photospheric magnetic field. Flux pileup $B_{s}$ can be large in a dense and cool photospheric plasma where the gas pressure significantly exceeds the magnetic pressure and the dimensionless electric resistivity can be of order $\eta \simeq 10^{-3}$. Flux pile-up merging has been shown to be sufficiently rapid to explain the observed cancellation rates, implying the reconnection inflow speeds that are a significant fraction of the local Alfvén speed, $v_{i} \simeq 0.1 v_{A}$ (Litvinenko et al. 2007).
As a simple illustration of a possible geometry of photospheric cancellation in a curved current sheet, consider two canceling magnetic fragments and suppose that they are separated by a sheet the cross-section of which can be approximated by a circular arc of length $L=\phi a$, where $\phi$ is the central angle, and $a$ is the radius of the circle. Suppose also that an observed inflow speed $v_{i}$ in a canceling magnetic feature is associated with the maximum speed reached on the chord joining the ends of the arc. Then using the observed values of $v_{i}, a$, and $L$ yields an expression for the model parameter $\alpha$ :

$$
\alpha=\frac{v_{i}}{a} \frac{\cos (L / 2 a)}{\sin ^{2}(L / 2 a)} .
$$

Furthermore, for a given resistivity $\eta$, the thickness of the sheet is predicted to scale as

$$
\frac{l}{a} \approx\left[\frac{\eta}{2 a v_{i}} \frac{\sin ^{2}(L / 2 a)}{\cos (L / 2 a)}\right]^{1 / 2} .
$$

These scalings could be used to constrain the parameters of the flux pile-up merging model of canceling magnetic features.

A potential difficulty with an incompressible model of this sort is that the solar atmosphere is actually highly stratified. The photosphere separates low-beta and high-beta plasma regions. Litvinenko (1999) addressed this concern by arguing that the vertical extent of a photospheric current sheet should be limited by the atmospheric pressure scale height, implying that the neglect of $z$-dependence of the magnetic field and density in the sheet could be justified to a reasonable degree of accuracy. The possibility of a time-dependent height of the reconnection site is discussed in the next section.

\section{Location of a Current Sheet in the SOlar ATMOSPHERE}

Park \& Chae (2012) used Hinode data to analyze transient Ca II H brightenings associated with canceling magnetic features in the quiet Sun. The observed association of transient $\mathrm{Ca}$ II $\mathrm{H}$ brightenings and canceling magnetic features supports chromospheric magnetic reconnection as the mechanism of both flux cancellation and plasma heating. The observations, however, appear to disagree with a theoretical model of the relationship between the atmospheric heating and canceling features.

Priest et al. (1994) modeled the cancellation geometry of two opposite-polarity magnetic fragments by two line sources with a superimposed uniform horizontal field. The resulting planar magnetic field has a simple analytical form, when written with the help of complex variables:

$$
B_{y}+i B_{x}=\frac{i f}{\pi(z-b)}-\frac{i f}{\pi(z+b)}+i B_{0},
$$

where $f$ and $B_{0}$ are parameters of the model, $2 b$ is the distance between the line sources, $z=x+i y$, and $y$ is the height above the photosphere. (Note for clarity that 


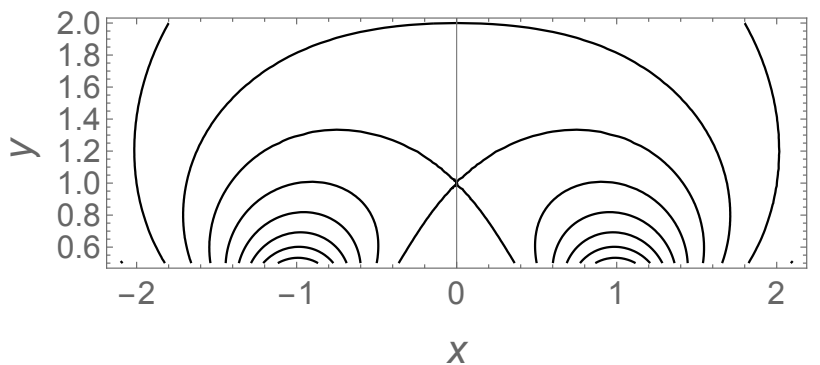

Figure 1. Magnetic field lines of two line dipoles, located at $(x, y)=(-b, 0)$ and $(x, y)=(b, 0)$. The parameter $b=1$. A magnetic null is located at $(x, y)=(0, b)$.

the notation differs somewhat from that of the previous section.) For a sufficiently slowly changing distance between the fragments, cancellation can be approximately modeled as a sequence of magnetostatic configurations rather than a magnetohydrodynamic process. Consequently the approach of the two fragments can be modeled by assuming a decreasing function of time $b=b(t)$.

As the two sources approach one another, a magnetic null appears at the photosphere $(y=0)$ when $b=2 f /\left(\pi B_{0}\right)$. As $b(t)$ continues to decrease, the null moves up and then down, and so reconnection-related plasma heating is predicted to occur in the solar corona before the sources come into contact. Consequently, a transient brightening should occur before the photospheric cancellation takes place. Observations, however, demonstrate the opposite behavior: the Ca II $\mathrm{H}$ intensity increases when the magnetic fluxes of the interacting fragments are already significantly reduced (Park \& Chae 2012).

The disagreement between the observations and the theory can be resolved by modifying the assumed geometry of the canceling magnetic fields. As an illustration, consider the magnetic field in a plane normal to two parallel line dipoles of equal moments, which slowly approach one another. Suppose for simplicity that the background magnetic field is negligibly weak. Then the model leads to the following expression for the planar magnetic field:

$$
B_{y}+i B_{x}=\frac{i D}{(z-b)^{2}}+\frac{i D}{(z+b)^{2}} .
$$

Here $2 b$ is the distance between the approaching dipoles, so that $b=b(t)$ is a slowly decreasing function of time $t$, and $D$ quantifies the dipole moment. Figure 1 shows the magnetic field lines, defined by the equation

$$
\frac{y}{(x-b)^{2}+y^{2}}+\frac{y}{(x+b)^{2}+y^{2}}=\text { const. }
$$

A magnetic null is located at height $y=b$ above the photosphere. Indeed, along the line $x=0$,

$$
B_{x}=2 D \frac{b^{2}-y^{2}}{\left(b^{2}+y^{2}\right)^{2}}, \quad B_{y}=0
$$

(Fig. 2). As the distance $2 b$ between the dipoles decreases, the null moves downwards. For a sufficiently

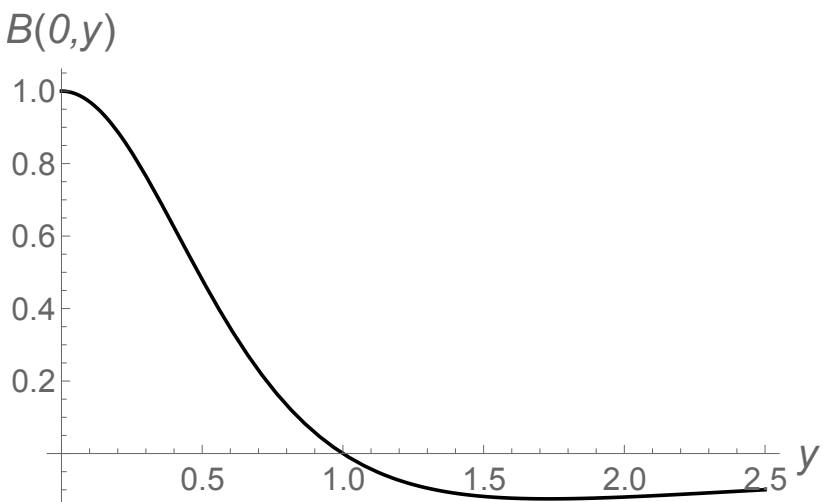

Figure 2. Magnetic field $B_{x}(0, y)$ between two canceling dipoles. The parameters are $D=0.5, b=1$.

small $b(t)$, reconnection starts in the chromosphere, and so the plasma heating rate and the associated radiation intensity should increase as cancellation proceeds, as observed. A more realistic description of the location of the observed brightening can be achieved by relaxing the assumption of equal dipole moments (see also Tur \& Priest 1976).

It may be useful to summarize the arguments that support magnetic reconnection in a relatively dense and cool chromospheric current sheet as the local heating process that causes the observed transient brightenings. For a chromospheric current sheet, a characteristic cooling time due to thermal conduction is too long in comparison with the cancellation time, implying that radiation is the dominant mechanism of energy loss from the sheet (Litvinenko \& Somov 1994). A characteristic conduction time (in seconds) can be estimated as

$$
\tau_{c} \simeq 4 \times 10^{-10} \frac{n L^{2}}{T^{5 / 2}}
$$

where $L$ is a characteristic length (in $\mathrm{cm}$ ), $n$ density (in $\mathrm{cm}^{-3}$ ), and $T$ temperature (in K) (Boyd \& Sanderson 1969). Assuming $L \simeq 10^{8} \mathrm{~cm}, n \simeq 10^{15} \mathrm{~cm}^{-3}$, and $T \simeq 1.5 \times 10^{4} \mathrm{~K}$ yields $\tau_{c} \simeq 10^{10} \mathrm{~s}$. Except for very small canceling features, conduction is too slow compared with a typical cancellation time.

The high density and low temperature in the chromospheric current sheet also mean that particle acceleration by the reconnection electric field is inefficient. Hence magnetic energy release by reconnection should lead to bulk plasma heating, balanced by radiation. A quantitative measure of the acceleration efficiency is the ratio of the reconnection electric field $E_{r}$ and the Dreicer electric field $E_{D}$. Typical values of the magnetic field $B_{i} \simeq 200 \mathrm{G}$ and plasma speed $v_{i} \simeq 100 \mathrm{~m} / \mathrm{s}$ in the reconnection inflow region (Litvinenko et al. 2007) yield

$$
E_{r}=\frac{1}{c} v_{i} B_{i} \simeq 10^{-4} \text { statvolt } / \mathrm{cm},
$$

where $c$ is the speed of light. Now, typical values of density $n \simeq 10^{15} \mathrm{~cm}^{-3}$ and temperature $T \simeq 1.5 \times 10^{4}$ 
$\mathrm{K}$ in the sheet yield

$$
E_{D}=\frac{4 \pi n e^{3}}{k T} \ln \Lambda \simeq 1 \text { statvolt } / \mathrm{cm},
$$

where $e$ is the electron charge, $k$ is the Boltzmann constant, and $\ln \Lambda$ is the Coulomb logarithm. Thus $E_{r} / E_{D} \ll 1$, which rules out efficient electron acceleration in a chromospheric current sheet.

Finally, in addition to bulk heating of the chromospheric plasma, reconnection converts a part of the free magnetic energy into the kinetic energy of reconnection jets. The jets travel with the Alfvén speed $v_{A}$. For the parameters above,

$$
v_{A}=\frac{B_{i}}{\sqrt{4 \pi m_{p} n}} \simeq 10^{6} \mathrm{~cm} / \mathrm{s}=10 \mathrm{~km} / \mathrm{s},
$$

where $m_{p}$ is the proton mass. Interestingly, the New Solar Telescope data revealed upward-propagating shock waves with speeds up to $70 \mathrm{~km} / \mathrm{s}$, associated with flux cancellation (Yang et al. 2014), implying that the speed of a reconnection jet can increase significantly as it travels upward through the solar atmosphere and the density of the surrounding plasma decreases. Assuming that magnetic tension in the reconnection outflow region further accelerates the jet, an upper limit on the jet speed could be determined by an interaction of the velocity and magnetic fields in a viscous boundary layer (Galloway et al. 1977, 1978; Parker 1982). Development of a quantitative model of the process is an interesting topic for further study.

\section{ACKNOWLedgments}

Useful comments and suggestions by the referee are gratefully acknowledged.

\section{REFERENCES}

Boyd, T. J. M., \& Sanderson, J. J. 1969, Plasma Dynamics (New York: Barnes \& Noble)

Chae, J. 2012, Magnetic Reconnection in the Photosphere and Chromosphere, in 4th Hinode Science Meeting: Unsolved Problems and Recent Insights, ASP Conference Series, 455, ed. L. R. Bellot Rubio, F. Reale, \& M. Carlsson (San Francisco, CA: ASP), 187

Chae, J., Moon, Y.-J., Wang, H., \& Yun, H. S. 2002, Flux Cancellation Rates and Converging Speeds of Canceling Magnetic Features, Solar Physics, 207, 73

Craig, I. J. D., \& Henton, S. M. 1995, Exact Solutions for Steady State Incompressible Magnetic Reconnection, ApJ, 450, 280
Galloway, D. J., Proctor, M. R. E., \& Weiss, N. O. 1977, Formation of Intense Magnetic Fields Near the Surface of the Sun, Nature, 266, 686

Galloway, D. J., Proctor, M. R. E., \& Weiss, N. O. 1978, Magnetic Flux Ropes and Convection, J. Fluid Mechanics, 87,243

Litvinenko, Y. E. 1999, Photospheric Magnetic Reconnection and Canceling Magnetic Features on the Sun, ApJ, 515,435

Litvinenko, Y. E. 2013, An Exact Solution for Magnetic Annihilation in a Curved Current Sheet, ApJ, 774, 155

Litvinenko, Y. E., Chae, J., \& Park, S.-Y. 2007, Flux Pile-up Magnetic Reconnection in the Solar Photosphere, ApJ, 662, 1302

Litvinenko, Y. E., \& Somov, B. V. 1994, On Magnetic Reconnection in the Temperature Minimum Region on the Sun, Astronomy Letters, 20, 185

Livi, S. H. B., Wang, J., \& Martin, S. F. 1985, The Cancellation of Magnetic Flux. II. On the Quiet Sun, Australian J. Phys., 38, 855

Martens, P. C., \& Zwaan, C. 2001, Origin and Evolution of Filament-Prominence Systems, ApJ, 558, 872

Martin, S. F. 1990, Small-Scale Magnetic Features Observed in the Photosphere, in IAU Symposium 138, Solar Photosphere: Structure, Convection, and Magnetic Fields, ed. J. O. Stenflo (Dordrecht: Kluwer), 129

Martin, S. F., Livi, S. H. B., \& Wang, J. 1985, The Cancellation of Magnetic Flux. I. In a Decaying Active Region, Australian J. Phys., 38, 929

Martin, S. F., Panasenco, O., Engvold, O., \& Lin, Y. 2008, The Link Between CMEs, Filaments and Filament Channels, Annales Geophysicae, 26, 3061

Panasenco, O., Martin, S. F., \& Velli, M. 2014, Apparent Solar Tornado-Like Prominences, Solar Physics, 289, 603

Park, S., \& Chae, J. 2012, Ca II Transient Brightenings Associated with Canceling Magnetic Features, Solar Physics, 280, 103

Park, S., Chae, J., \& Litvinenko, Y. E. 2009, Rates of Photospheric Magnetic Flux Cancellation Measured with Hinode, ApJL, 704, L71

Parker, E. N. 1982, Compression of Magnetic Field in a Viscous Boundary Layer, Solar Physics, 77, 3

Priest, E. R., Parnell, C. E., \& Martin, S. F. 1994, A Converging Flux Model of an X-ray Bright Point and an Associated Canceling Magnetic Feature, ApJ, 427, 459

Tur, T. J., \& Priest, E. R. 1976, The Formation of Current Sheets During the Emergence of New Magnetic Flux from Below the Photosphere, Solar Physics, 48, 89

Yang, H., Chae, J., Lim, E.-K., Lee, K.-S., Park, H., Song, D.-U., \& Cho, K. 2014, Magnetic-Reconnection Generated Shock Waves as a Driver of Solar Surges, ApJL, 790, L4

Zeng, Z., Cao, W., \& Ji, H. 2013, Observation of Magnetic Reconnection Driven by Granular Scale Advection, ApJL, 790, L4 